\title{
openheart Differences of in-hospital outcomes within patients undergoing percutaneous coronary intervention at institutions with high versus low procedural volume: a report from the Japanese multicentre percutaneous coronary intervention registry
}

\author{
Masaki Kodaira, ${ }^{1}$ Toshiki Kuno, ${ }^{1}$ Yohei Numasawa, ${ }^{1}$ Takahiro Ohki, ${ }^{2}$ \\ Iwao Nakamura, ${ }^{3}$ Ikuko Ueda, ${ }^{4}$ Keiichi Fukuda, ${ }^{4}$ Shun Kohsaka ${ }^{4}$
}

\begin{abstract}
- Additional material is published online only. To view please visit the journal online (http://dx.doi.org/10.1136/ openhrt-2018-000781)
\end{abstract}

To cite: Kodaira M, Kuno T, Numasawa $Y$, et al. Differences of in-hospital outcomes within patients undergoing percutaneous coronary intervention at institutions with high versus low procedural volume: a report from the Japanese multicentre percutaneous coronary intervention registry. Open Heart 2018;5:e000781. doi:10.1136/ openhrt-2018-000781

Received 12 January 2018 Revised 17 April 2018 Accepted 29 May 2018

D Check for updates

${ }^{1}$ Department of Cardiology, Japanese Red Cross Ashikaga Hospital, Tochigi, Japan ${ }^{2}$ Department of Cardiology, Tokyo Dental College, Ichikawa General Hospital, Chiba, Japan ${ }^{3}$ Department of Cardiology, Hino Shiritsu Byoin, Tokyo, Japan ${ }^{4}$ Department of Cardiology, Keio University School of Medicine, Tokyo, Japan

Correspondence to Dr Masaki Kodaira; mskodaira@ gmail.com

\section{ABSTRACT}

Objective We aimed to determine the relationship between the prevalence of in-hospital complications and annual institutional patient volume in a population of patients undergoing percutaneous coronary intervention (PCl). Methods Clinical data of patients receiving $\mathrm{PCl}$ between January 2010 and June 2015 were collected from 14 academic institutions in the Tokyo area and subsequently used for analysis. We employed multivariate hierarchical logistic regression models to determine the effect of institutional volume on several in-hospital outcomes, including in-hospital mortality and procedure-related complications.

Results A total of $14437 \mathrm{PCl}$ cases were included and categorised as receiving intervention from either lowervolume ( $<200$ procedures/year, $n=6$ hospitals) or highervolume ( $\geq 200$ procedures/year, $n=8$ hospitals) institutions. Clinical characteristics differed significantly between the two patient groups. Specifically, patients treated in highervolume hospitals presented with increased comorbidities and complex coronary lesions. Unadjusted mortality and complication rate in lower-volume and higher-volume hospitals were $1.3 \%$ and $1.2 \%(p=0.0614)$ and $6.2 \%$ and $8.1 \%(p=0.001)$, respectively. However, multivariate hierarchical logistic regression models adjusting for differences in the patient characteristics demonstrated that institutional volume was not associated with adverse clinical outcomes.

Conclusions In conclusion, we observed no significant association between annual institutional volume and in-hospital outcomes within the contemporary PCl multicentre registry.

Trial registration number UMIN R000005598.

\section{INTRODUCTION}

Despite reduction in mortality over the past decade, ${ }^{1}$ cardiovascular disease remains a leading cause of death worldwide. ${ }^{2-4}$

\section{Key questions}

What is already known about this subject?

- Multiple reports have shown an inverse association between institutional volume and outcomes in patients undergoing percutaneous coronary intervention $(\mathrm{PCl})$.

What does this study add?

- This was a large-sample observational study of patients who received PCls performed in Japan.

- This study was designed to evaluate the association between institutional volume and in-hospital mortality or complications in the modern drug-eluting stent era.

- The study's data were obtained from a prospective multicentre registry, collecting 200 clinical variables and outcomes, of which we were able to investigate.

- Multivariate hierarchical logistic regression models revealed that institutional volume was not associated with any of the clinical outcomes in Japan.

How might this impact on clinical practice?

- The magnitude of the volume-outcome relationship could be modest for $\mathrm{PCl}$ in the modern era because it has become a common procedure.

Interestingly, several studies report significant variability in the clinical performance of therapeutic modalities such as percutaneous coronary intervention (PCI). ${ }^{56}$ Specifically, numerous studies demonstrate that greater institutional volume is associated with improved patient outcomes following PCI. ${ }^{7-10}$ Conversely, multiple reports consistently associate centres performing less than 200 PCIs per year with significantly poorer patient outcomes. ${ }^{1011}$ Accordingly, the 2013 
American College of Cardiology Foundation/American Heart Association/Society for Cardiovascular Angiography and Interventions clinical competence statement on coronary artery procedures recommends that PCIs be performed at high-volume centres. ${ }^{12}$ However, studies performed in Taiwan and Japan, where the majority of the population is covered by health insurance ${ }^{13}$ and the incidence and mortality rate due to coronary heart disease is relatively low, ${ }^{14-18}$ demonstrate no significant relationship between institutional volume and clinical outcome. ${ }^{19-21}$

As a rather large proportion of PCI centres in Japan still perform less than 200 PCIs annually, ${ }^{22}{ }^{23}$ it is of critical importance to determine if and to what extent institutional volume is associated with clinical outcome. Therefore, the objective of our investigation was to (1) compare clinical characteristics, treatment patterns and adherence to guidelines on medication between low-volume and high-volume PCI centres and (2) evaluate the association between institutional volume and in-hospital adverse outcomes using the Japan Cardiovascular Database-Keio inter-hospital Cardiovascular Studies (JCD-KiCS) PCI registry.

\section{METHODS}

\section{Data source}

Patient cohorts were derived from the JCD-KiCS database. The JCD-KiCS is a prospective, multicentre registry developed to archive clinical data on patients undergoing PCI. Clinical variables and in-hospital outcomes of the JCD-KiCS were defined in accordance with the large PCI registry in the USA, National Cardiovascular Data Registry (NCDR) V.4.1. ${ }^{24} 25$ The study was approved by the institutional review board of Keio University School of Medicine. Before the launch of the registry, information on the objectives of the present study, its social significance and an abstract were provided for clinical trial registration with the University Hospital Medical Information Network. Details of JCD-KiCS data collection and audit processes have previously been described. ${ }^{26-28}$

\section{Study population}

Between January 2010 and June 2015, 15125 PCIs were performed at 15 hospitals in the JCD-KiCS PCI registry (figure 1). A total of six and eight hospitals were assigned to the lower-volume and higher-volume hospital groups, respectively. A total of 633 cases were excluded due to presentation of cardiogenic shock on arrival. Additionally, 55 cases performed in one of the hospitals were excluded as this hospital discontinued registration after March 2011. Annual institutional volume was calculated for each hospital from its total number of PCIs registered in JCD-KiCS during the study period, divided by the number of years during which the number of PCI procedures was non-zero, based on the unique hospital identification number. The patient population was then subdivided into two hospital groups based on annual

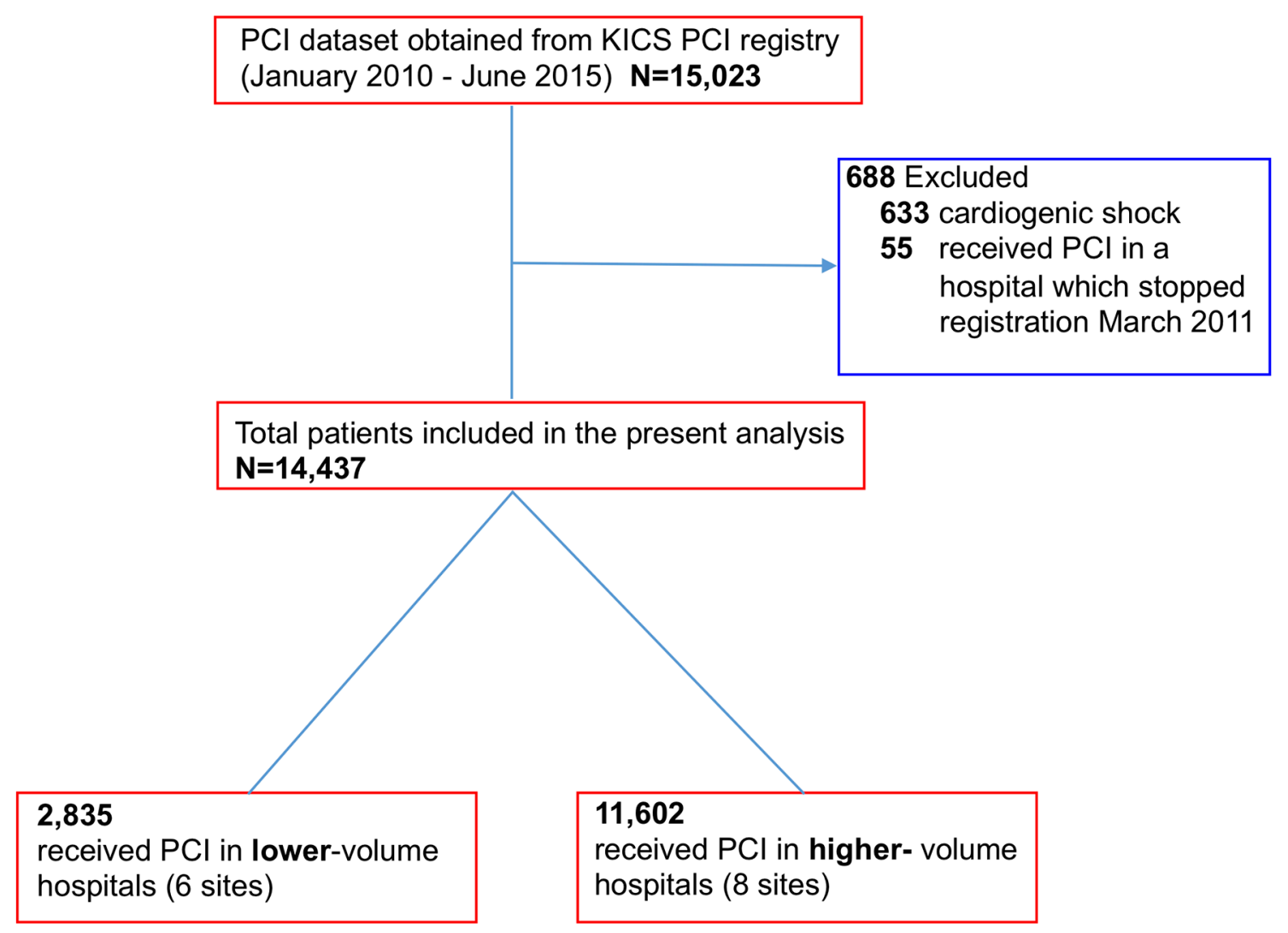

Figure 1 Flowchart of the study population. KiCS, Keio inter-hospital Cardiovascular Studies; PCl, percutaneous coronary intervention. 
institutional volume: <200 cases/year (lower volume) and $\geq 200$ cases/year (higher volume).

\section{Study endpoints}

The primary outcome identified was the occurrence of in-hospital mortality; secondary outcomes were general in-hospital complications. General complications were defined as the occurrence of one or more of the following procedural complications: severe coronary dissection or perforation; myocardial infarction after PCI; cardiac shock or heart failure; cerebral bleeding or stroke; bleeding complications. Bleeding was defined as the presence of one or both of the following within 72 hours of PCI: requirement of blood transfusion or prolongation of hospital stay with decreases in haemoglobin more than $3.0 \mathrm{~g} / \mathrm{dL}$. These eventualities are equivalent to a type 3 classification according to Bleeding Academic Research Consortium criteria. ${ }^{29}$ Furthermore, bleeding complications were classified into puncture-site bleeding, haematoma, retroperitoneal bleeding, gastrointestinal bleeding, genitourinary bleeding or other bleeding.

\section{Statistical analysis}

Results are reported as mean $\pm \mathrm{SD}$ for continuous variables and as a percentage for categorical variables. Differences between the two cohorts were analysed using $\chi^{2}$ test for categorical variables and Student's t-test for continuous variables. For missing adjustment variables, multiple imputations were used. Hierarchical modelling was purposefully designed to analyse data with nested observations. Multilevel hierarchical mixed-level logistic regression modelling ${ }^{30}$ (with patient-level factors nested within hospital-level factor) was used with hospital number as a random effect to compare in-hospital mortality and complication rates between lower-volume and higher-volume hospitals. Variables used for adjustment include patient characteristics from the CathPCI risk model. ${ }^{31}$ The analyses were adjusted for age, heart failure at admission, presentation with ST-segment elevation myocardial infarction (STEMI), institutional volume level (higher: annual volume $\geq 200$ cases per year; lower: annual volume $<200$ cases per year), peripheral vascular disease, prior heart failure, estimated glomerular filtration rate and chronic lung disease. We further performed subgroup analyses by including interaction between institutional volume and the variable of interest. Sensitivity analysis was performed incorporating year of PCI as variable for multiple logistic regression. Sensitivity analysis was also performed using continuous variable for institutional volume. SPSS V.24.0 (IBM) was used for analysis and statistical significance was set at $p<0.05$. For comparison of various unadjusted in-hospital outcomes, we applied Bonferroni adjustment to address the problem associated with multiple comparisons. We divided the alpha level $(0.05)$ by the number of in-hospital outcomes being compared (10) and used the computed value, 0.005 , as the new alpha for this analysis.
Table 1 Baseline characteristics by annual hospital volume

$\begin{array}{ll}\begin{array}{l}\text { Lower volume } \\ \text { (<200 procedures/ }\end{array} & \begin{array}{l}\text { Higher volume } \\ (\geq 200 \text { procedures/ } \\ \text { year) }\end{array} \\ (n=2835) & \text { year) } \\ & (n=11602)\end{array}$

\begin{tabular}{|c|c|c|}
\hline \multicolumn{3}{|l|}{ Demographics } \\
\hline Female & $611(22.8 \%)$ & $2329(21.1 \%)$ \\
\hline Age & $67.4 \pm 11.0$ & $67.8 \pm 11.1$ \\
\hline BMI & $24.4 \pm 3.6$ & $24.1 \pm 3.6$ \\
\hline \multicolumn{3}{|l|}{ Medical history } \\
\hline Diabetes (\%) & 1067 (37.6\%) & $4658(40.1 \%)$ \\
\hline Hypertension (\%) & 2016 (71.1\%) & 8202 (70.7\%) \\
\hline Smoker (\%) & $1021(36.0 \%)$ & 3471 (29.9\%) \\
\hline Dyslipidaemia (\%) & $1701(60.0 \%)$ & $7328(63.2 \%)$ \\
\hline $\begin{array}{l}\text { Chronic renal } \\
\text { failure (\%) }\end{array}$ & $976(39.6 \%)$ & $4621(42.5 \%)$ \\
\hline Dialysis (\%) & $116(4.1 \%)$ & $551(4.7 \%)$ \\
\hline $\begin{array}{l}\text { Chronic lung } \\
\text { disease (\%) }\end{array}$ & $71(2.5 \%)$ & $355(3.1 \%)$ \\
\hline $\begin{array}{l}\text { Peripheral vascular } \\
\text { disease (\%) }\end{array}$ & $146(5.1 \%)$ & $1059(9.1 \%)$ \\
\hline $\begin{array}{l}\text { Previous heart } \\
\text { failure (\%) }\end{array}$ & $211(7.4 \%)$ & 1029 (8.9\%) \\
\hline History of MI (\%) & 645 (22.8\%) & 2637 (22.7\%) \\
\hline $\begin{array}{l}\text { Cerebral artery disease } \\
(\%)\end{array}$ & $186(6.6 \%)$ & 1017 (8.8\%) \\
\hline History of PCl (\%) & 988 (34.9\%) & $4243(36.6 \%)$ \\
\hline \multicolumn{3}{|l|}{ Clinical presentation } \\
\hline STEMI (\%) & $630(22.2 \%)$ & 2147 (18.5\%) \\
\hline NSTEMI (\%) & 201 (7.1\%) & 818 (7.1\%) \\
\hline $\begin{array}{l}\text { Unstable } \\
\text { angina (\%) }\end{array}$ & 485 (17.1\%) & $2059(17.7 \%)$ \\
\hline $\begin{array}{l}\text { Heart failure } \\
\text { symptom (\%) }\end{array}$ & $348(12.3 \%)$ & $266(10.9 \%)$ \\
\hline
\end{tabular}

Values are presented as $\mathrm{n}(\%)$ or mean $\pm \mathrm{SD}$, as indicated. BMI, body mass index; MI, myocardial infarction; NSTEMI, non-ST-elevation myocardial infarction; $\mathrm{PCl}$, percutaneous coronary intervention; STEMI, ST-elevation myocardial infarction.

\section{RESULTS}

\section{Baseline characteristics of the study population}

In total, 14437 PCI cases met the specified enrolment criteria. With respect to annual volume, 2835 cases were performed in lower-volume hospitals and 11602 cases in higher-volume hospitals. Baseline patient demographic and clinical features stratified by annual institutional volume are listed in table 1 . Interestingly, several parameters were significantly different between the two patient cohorts. Specifically, patients treated in lowervolume hospitals were more likely to smoke and present with STEMI. Further, comorbidities such as diabetes, renal dysfunction, dialysis, dyslipidaemia, cerebral artery disease and peripheral vascular disease were less prevalent in patients treated at low-volume institutions. 
Table 2 Procedural characteristics by annual hospital volume

\begin{tabular}{|c|c|c|}
\hline & $\begin{array}{l}\text { Lower volume: } \\
<200 \\
\text { procedures/ } \\
\text { year } \\
(\mathrm{n}=2835)\end{array}$ & $\begin{array}{l}\text { Higher volume: } \\
\geq 200 \text { procedures/ } \\
\text { year } \\
(n=11602)\end{array}$ \\
\hline \multicolumn{3}{|l|}{ Diseased vessels } \\
\hline $\begin{array}{l}\text { Three-vessel } \\
\text { disease (\%) }\end{array}$ & $688(24.3 \%)$ & $2575(22.2 \%)$ \\
\hline $\begin{array}{l}\text { Left main trunk } \\
\text { lesion }(\%)\end{array}$ & $105(3.7 \%)$ & $532(4.6 \%)$ \\
\hline \multicolumn{3}{|l|}{ Location of target } \\
\hline Left main trunk & $64(2.3 \%)$ & $421(3.6 \%)$ \\
\hline $\begin{array}{l}\text { Left anterior } \\
\text { descending }\end{array}$ & $1560(45.0 \%)$ & $4960(42.8 \%)$ \\
\hline Right & $912(32.2 \%)$ & $3556(30.6 \%)$ \\
\hline \multicolumn{3}{|l|}{ Highest-risk lesion } \\
\hline $\begin{array}{l}\text { ACC/AHA type C } \\
\text { lesion (\%) }\end{array}$ & $629(22.2 \%)$ & 3755 (32.4\%) \\
\hline $\begin{array}{l}\text { Bifurcation } \\
\text { lesion (\%) }\end{array}$ & $792(27.9 \%)$ & $3273(28.2 \%)$ \\
\hline CTO (\%) & $139(4.9 \%)$ & $832(7.2 \%)$ \\
\hline \multicolumn{3}{|l|}{ Type of stent } \\
\hline DES (\%) & $1685(59.4 \%)$ & $8293(71.5 \%)$ \\
\hline \multicolumn{3}{|l|}{ Access site } \\
\hline TRI (\%) & $550(19.4 \%)$ & $5212(44.9 \%)$ \\
\hline \multicolumn{3}{|l|}{ Device } \\
\hline IABP (\%) & $91(3.2 \%)$ & $611(5.3 \%)$ \\
\hline IVUS (\%) & $2379(83.9 \%)$ & $10158(87.6 \%)$ \\
\hline $\begin{array}{l}\text { Amount of contrast } \\
\text { medium }(\mathrm{mL})\end{array}$ & $206.0 \pm 96.1$ & $162.5 \pm 71.2$ \\
\hline
\end{tabular}

Values are presented as $\mathrm{n}(\%)$ or mean $\pm \mathrm{SD}$, as indicated. ACC, American College of Cardiology; AHA, American Heart Association; CTO, chronic total occlusion; DES, drug-eluting stent; IABP, intra-aortic balloon pump; IVUS, intravascular ultrasound; TRI, trans-radial intervention.

Conversely, patients treated in higher-volume hospitals were comparatively older.

\section{Procedural characteristics}

Table 2 compares the procedural characteristics across the two patient cohorts. Patients treated in lowervolume hospitals were statistically less likely to receive a drug-eluting stent. Further, less than $20 \%$ of patients at lower-volume hospitals received PCI via radial access, significantly fewer compared with higher-volume centres ( $\sim 5 \%$ of PCI cases). In contrast, patients treated in higher-volume hospitals required intra-aortic balloon pump support at a significantly higher frequency compared with lower-volume treatment facilities. Lastly, prescription rates of cardiac medication in relation to annual hospital volume are shown in table 3 . Notably, statin prescription
Table 3 Prescription rate of cardiac medication by annual hospital volume

\begin{tabular}{lll}
\hline & $\begin{array}{l}\text { Lower volume: } \\
<\mathbf{2 0 0} \\
\text { procedures/ } \\
\text { year } \\
\text { (n=2835) }\end{array}$ & $\begin{array}{l}\text { Higher volume: } \\
\mathbf{2 0 0} \\
\text { procedures/ } \\
\text { year } \\
(\mathbf{n = 1 1 6 0 2 )}\end{array}$ \\
\hline Clopidogrel at arrival (\%) & $2260(79.7 \%)$ & $9291(80.1 \%)$ \\
\hline DAPT at arrival (\%) & $2335(82.4 \%)$ & $9719(83.8 \%)$ \\
\hline Clopidogrel at discharge (\%) & $2525(89.1 \%)$ & $10324(89.0 \%)$ \\
\hline DAPT at discharge (\%) & $2608(92.0 \%)$ & $10717(92.4 \%)$ \\
\hline $\begin{array}{l}\text { Beta-blocker at } \\
\text { discharge (\%) }\end{array}$ & $1794(66.7 \%)$ & $7343(66.0 \%)$ \\
\hline Statin at discharge (\%) & $2138(75.4 \%)$ & $9141(78.8 \%)$ \\
\hline
\end{tabular}

Values are presented as $\mathrm{n}(\%)$.

DAPT, dual antiplatelet therapy.

rate was significantly higher for patients receiving care at higher-volume hospitals.

\section{Unadjusted in-hospital outcomes}

Figure 2 shows the comparison of both in-hospital mortality and in-hospital complications between the patient cohorts. Overall, in-hospital mortality rates were similar between the two groups: $1.3 \%$ and $1.2 \%$ ( $p=0.0614$; Bonferroni correction $\mathrm{p}<0.005)$ for low-volume and high-volume hospitals, respectively. Interestingly, the frequency of general in-hospital complications was significantly lower among patients treated in low-volume hospitals $(6.2 \%$ and $8.1 \%, \mathrm{p}=0.001$; Bonferroni correction $\mathrm{p}<0.005)$. Further, the frequencies of bleeding events within 72 hours $(1.3 \%$ and 2.4\%, $\mathrm{p}<0.001$; Bonferroni correction $\mathrm{p}<0.005$ ) and transfusion $(1.1 \%$ and $1.9 \%, \mathrm{p}=0.004$; Bonferroni correction $\mathrm{p}<0.005$ ) were all significantly reduced among patients treated in low-volume hospitals. However, after Bonferroni correction, incidence of post-PCI myocardial infarction $(0.9 \%$ and $1.6 \%, \mathrm{p}=0.006$; Bonferroni correction $\mathrm{p}<0.005)$ was no longer different between the two groups. Moreover, no significant difference was noted between the two patient cohorts with respect to incidence of post-PCI heart failure $(1.3 \%$ and $1.4 \%, \mathrm{p}=0.69$; Bonferroni correction $\mathrm{p}<0.005)$, post-PCI cardiogenic shock $(1.1 \%$ and $1.5 \%, \mathrm{p}=0.057$; Bonferroni correction $\mathrm{p}<0.005)$, post-PCI cerebral infarction $(0.3 \%$ and $0.3 \%$, $\mathrm{p}=0.923$; Bonferroni correction $\mathrm{p}<0.005)$, acute kidney injury $(13.7 \%$ and $13.5 \%, \mathrm{p}=0.769$; Bonferroni correction $\mathrm{p}<0.005)$ or new requirement for dialysis $(0.4 \%$ and $0.6 \%, \mathrm{p}=0.117$; Bonferroni correction $\mathrm{p}<0.005$ ).

\section{Predictors of patient mortality and general complications}

With respect to the overall patient population, in-hospital mortality was predicted by multiple variables including age, glomerular filtration rate, heart failure at admission and presence of STEMI (table 4). Importantly, our analysis demonstrated that institutional volume was not an independent predictor of in-hospital mortality. Further, variables 


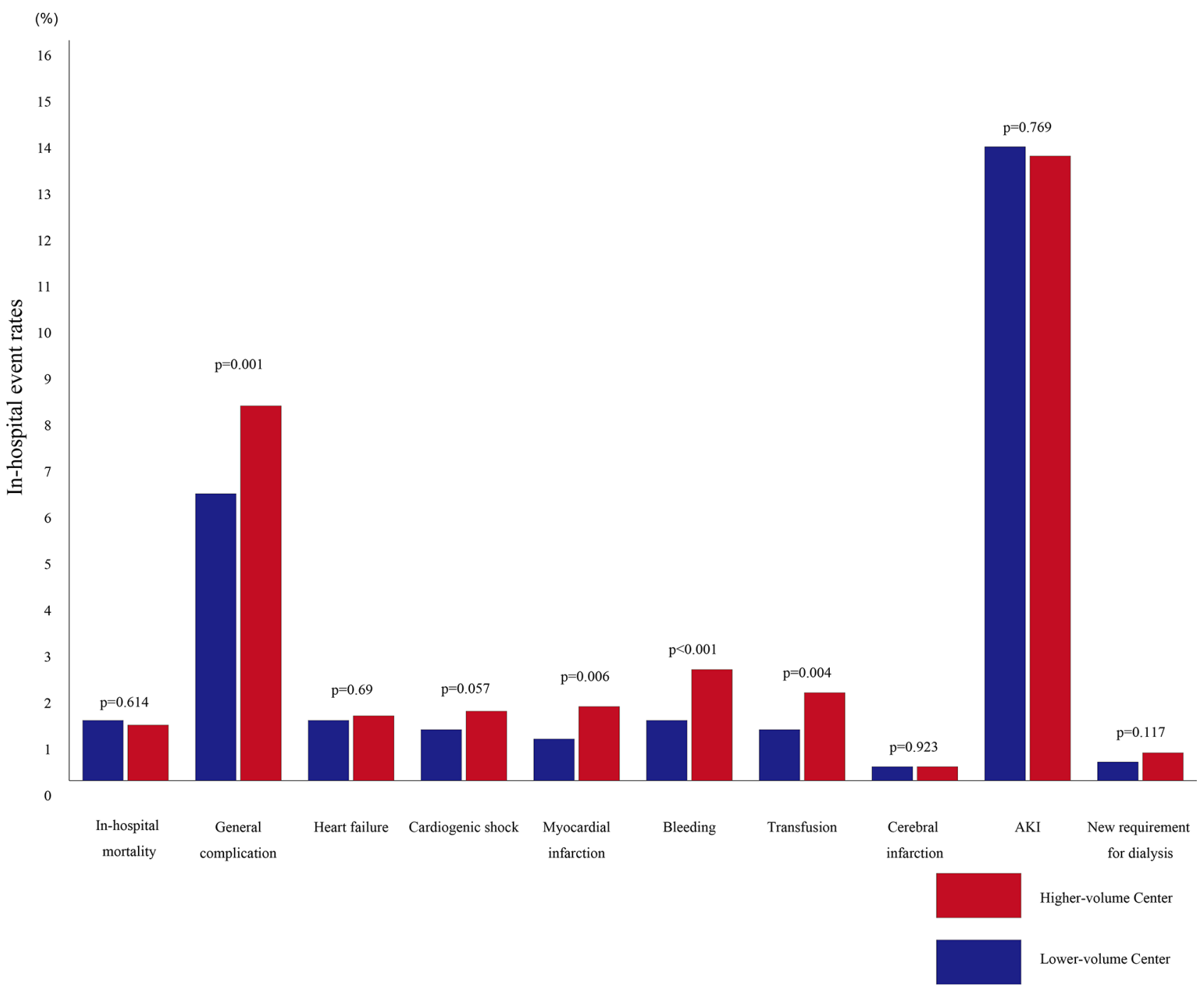

Figure 2 Rates of unadjusted in-hospital post- $\mathrm{PCl}$ clinical outcomes according to institutional volume. In-hospital mortality and complication rates per institutional volume groups are shown. Bonferroni correction by considering $\mathrm{p}<0.005$ for betweengroup differences in outcomes. AKI, acute kidney injury; PCI, percutaneous coronary intervention.

\begin{tabular}{|c|c|c|c|c|}
\hline & OR & $\begin{array}{l}\text { Lower } \\
95 \% \mathrm{Cl}\end{array}$ & $\begin{array}{l}\text { Upper } \\
95 \% \mathrm{Cl}\end{array}$ & $P$ values \\
\hline \multicolumn{5}{|l|}{ Hospital volume } \\
\hline Lower volume & Referent & Referent & Referent & \\
\hline Higher volume & 1.019 & 0.890 & 1.167 & 0.784 \\
\hline Prior heart failure & 1.221 & 0.979 & 1.522 & 0.076 \\
\hline Age & 1.010 & 1.004 & 1.016 & 0.002 \\
\hline GFR & 1.010 & 1.004 & 1.016 & 0.002 \\
\hline $\begin{array}{l}\text { Heart failure at } \\
\text { admission }\end{array}$ & 1.496 & 1.239 & 1.808 & $<0.001$ \\
\hline Chronic lung disease & 0.928 & 0.639 & 1.348 & 0.695 \\
\hline STEMI & 1.627 & 1.399 & 1.891 & $<0.001$ \\
\hline $\begin{array}{l}\text { Peripheral vascular } \\
\text { disease }\end{array}$ & 1.022 & 0.814 & 1.284 & 0.851 \\
\hline
\end{tabular}

GFR, glomerular filtration rate; STEMI, ST-elevation myocardial infarction. predicting general in-hospital complications included prior heart failure, age, glomerular filtration rate, heart failure at admission and presence of STEMI (table 5). Again, our analysis indicates that institutional volume did not independently predict general in-hospital complications.

\section{Subgroup and sensitivity analyses}

We found no significant difference in the magnitude of effect of institutional volume on in-hospital mortality or general complications according to whether the presentation was STEMI or not $(\mathrm{p}=0.42$ and $\mathrm{p}=0.12)$, whether the target lesion was type $\mathrm{C}$ or not $(\mathrm{p}=0.37$ and $\mathrm{p}=0.71)$, whether the target was chronic total occlusion or not ( $\mathrm{p}=0.86$ and $\mathrm{p}=0.27)$ and whether the target included bifurcation ( $\mathrm{p}=0.85$ and $\mathrm{p}=0.81$ ) (figures 3 and 4 ). In our sensitivity analysis, which incorporated the year of PCI as a time-related variable, we found no significant difference in the magnitude of effect of institutional volume on in-hospital mortality (OR 1.099, 95\% CI 0.777 to $1.555 ; \mathrm{p}=0.592)$ or general complications 


\begin{tabular}{|c|c|c|c|c|}
\hline & OR & $\begin{array}{l}\text { Lower } \\
95 \% \mathrm{Cl}\end{array}$ & $\begin{array}{l}\text { Upper } \\
95 \% \mathrm{Cl} \\
\end{array}$ & P values \\
\hline \multicolumn{5}{|l|}{ Hospital volume } \\
\hline Lower volume & Referent & Referent & Referent & \\
\hline Higher volume & 1.433 & 0.954 & 2.152 & 0.083 \\
\hline Prior heart failure & 1.339 & 1.150 & 1.558 & $<0.001$ \\
\hline Age & 1.020 & 1.015 & 1.025 & $<0.001$ \\
\hline GFR & 0.987 & 0.985 & 0.989 & $<0.001$ \\
\hline $\begin{array}{l}\text { Heart failure at } \\
\text { admission }\end{array}$ & 1.663 & 1.457 & 1.898 & $<0.001$ \\
\hline Chronic lung disease & 1.039 & 0.810 & 1.333 & 0.761 \\
\hline STEMI & 2.704 & 2.441 & 2.996 & $<0.001$ \\
\hline $\begin{array}{l}\text { Peripheral vascular } \\
\text { disease }\end{array}$ & 1.059 & 0.905 & 1.239 & 0.475 \\
\hline
\end{tabular}

GFR, glomerular filtration rate; STEMI, ST-elevation myocardial infarction.

(OR $1.407,95 \%$ CI 0.878 to 2.257; $\mathrm{p}=0.156$ ) (online supplementary tables S1 and S2). Although mortality rate remained stable throughout the study period, complication rates have significantly declined recently. Results were unchanged analysing institutional volume as continuous variable (online supplementary tables S2 and S4).

\section{DISCUSSION}

Our present investigation demonstrates three significant findings: First, the patients in the two analysis groups present with significantly different clinical and angiographic backgrounds. Second, although the two patient cohorts had similar in-hospital mortality, lowervolume hospitals were associated with significantly improved in-hospital outcomes with respect to postprocedural complications. Third and most importantly, after adjusting for confounding factors, institutional procedural volume was not associated with in-hospital mortality or general complications.

We observed wide variation in patient background and coronary lesion characteristics between the two patient cohorts. The patients receiving treatment at lower-volume hospitals exhibited significantly reduced comorbidity and were less likely to present with complex coronary lesions. Previous reports indicate that patients presenting with heart failure or multiple comorbidities are often treated with medical therapy alone. ${ }^{32} 33$ Moreover, Pandey et al demonstrated that annual PCI volume was significantly lower in patients with diabetes mellitus who were admitted with non-STEMI and did not receive revascularisation. ${ }^{32}$ Indeed, it is imperative to note that patient risk status may influence the current volumeoutcome theory. According to Lee and Kwak, ${ }^{34}$ obstetric delivery service at high-volume hospitals was associated with better patient outcomes in a defined 'medium-risk' group, while this volume-outcome relationship was not

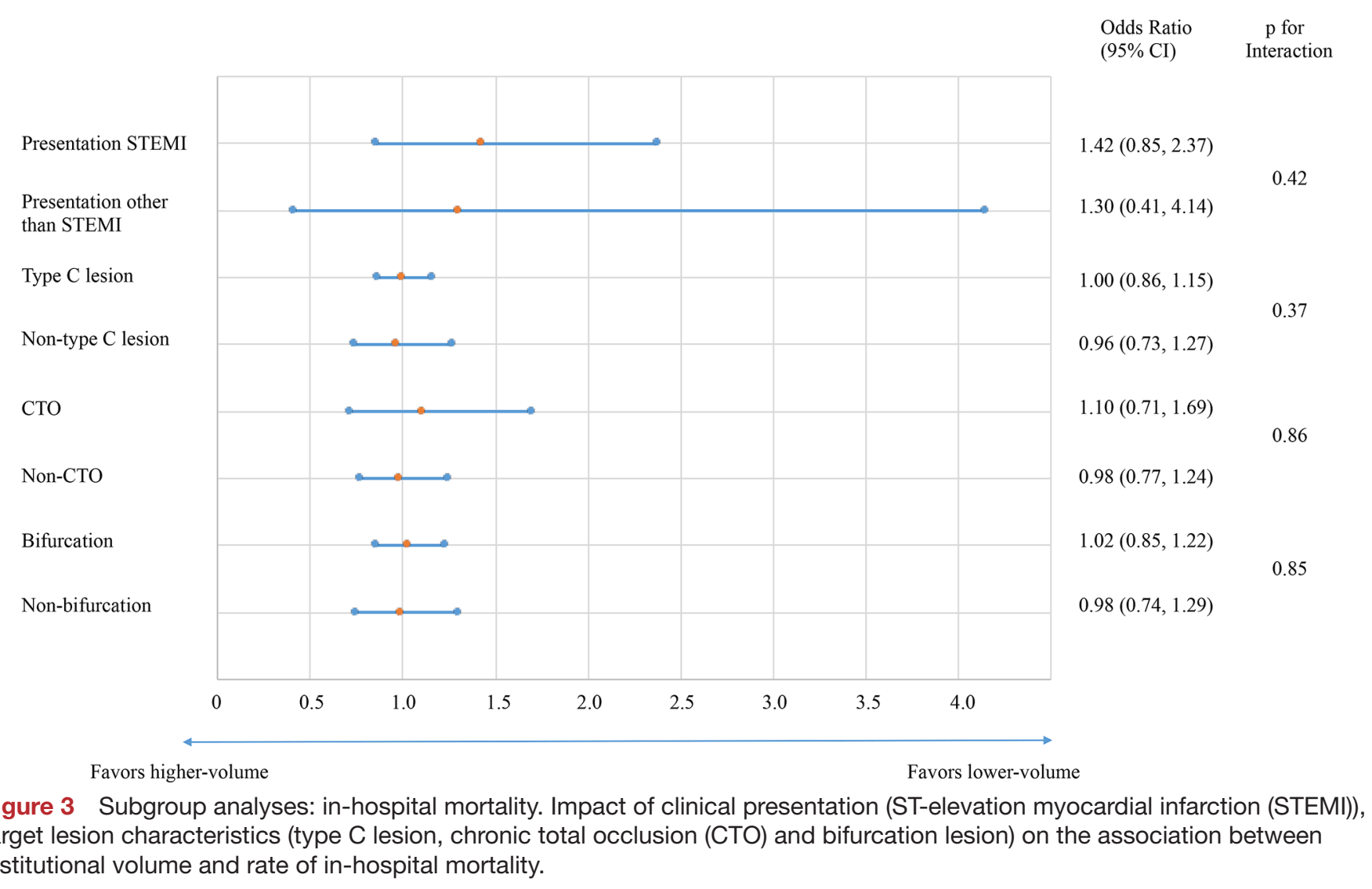




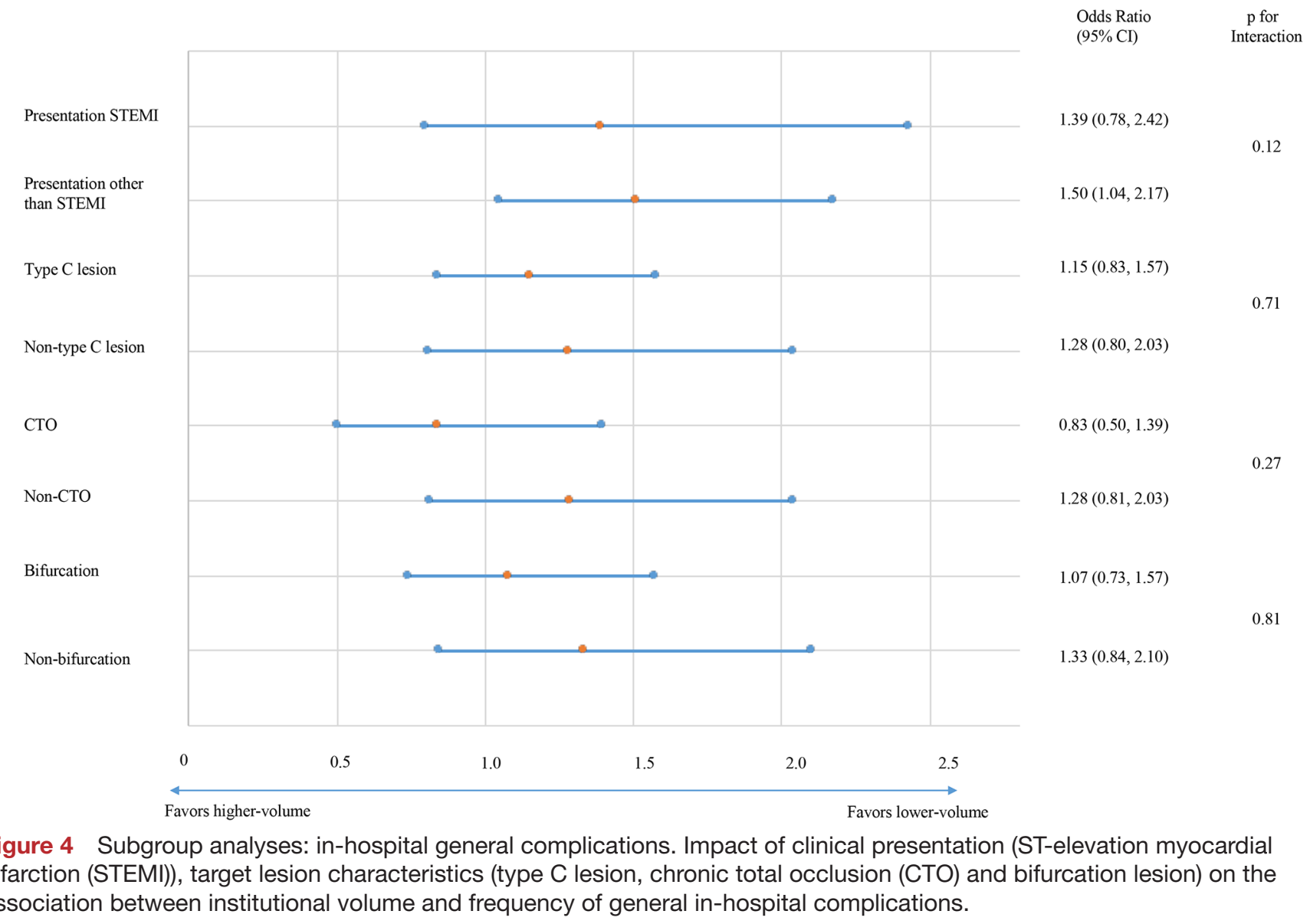

observed in relative low-risk or high-risk patient groups. Compared with previous reports, our patient population was composed of markedly higher-risk patients, with higher frequencies of elderly patients and patients with diabetes undergoing PCI. Further, the increased prevalence of high-risk patients may explain why a positive volume-outcome relationship was not identified in the present investigation.

Our data further indicate that unadjusted in-hospital complication rates were significantly increased at higher-volume institutions, a finding attributed to the increased incidence of bleeding events and procedural myocardial infarction. We previously reported that patients receiving PCI in Japan present with a significant prevalence of complex coronary lesions, including type $\mathrm{C}$, bifurcation and CTO. ${ }^{35}$ Interestingly, our analysis indicates an enhanced frequency of these coronary lesions in patients receiving intervention at higher-volume hospitals. Due to increased procedural experience, interventional cardiologists at highvolume care centres may have been more confident and, as a result, overly aggressive in treating these challenging coronary lesions. This tendency has been observed in multiple reports ${ }^{736}$ and appears pronounced in our investigation. Indeed, differences in local expertise have been reported to influence the decision-making process of clinical cardiologists. ${ }^{32}{ }^{37}$ As a result, the difference observed in the background and clinical risk factors between the two patient groups could have played a greater role than their technical differences.

Importantly, our analysis demonstrates that the risk-adjusted in-hospital mortality and complication rates were similar between lower-volume and higher-volume hospitals, regardless of lesion complexity. Compared with our investigation, a majority of previous studies predominantly focused on patients treated in hospitals with larger institutional volume. Genetic, anatomical, environmental and dietary differences have been suggested to influence variance in clinical outcome between different countries. ${ }^{38}$ Moreover, this heterogeneity between countries could be exacerbated by differences in the quality of medical care as well as financial support received by medical centres. ${ }^{39}$ In Japan, for example, the majority of medical expenses are covered by health insurance. ${ }^{13}$ Consequently, physicians are able to perform the required procedures, with little pressure from insurance companies regarding potential cost. ${ }^{40}$ Due to these differences, it is possible that associations between institutional volume and clinical outcome may not occur in these countries. Indeed, Lin $e t a l^{19}$ reported no significant difference in 30-day mortality between medium-volume hospitals (200-399 cases per year) and high-volume hospitals ( $\geq 400$ cases per year). Similarly, studies performed by Shiraishi $e t a t^{21}$ and Tsuchihashi $e t a t^{20}$ found no significant difference in in-hospital mortality rates between highvolume hospitals and low-volume hospitals among patients 
undergoing PCI for acute myocardial infarction. Recently, Inohara et al demonstrated the association between lower institutional volume $(<150$ cases per year $)$ and worse in-hospital outcomes in nationwide Japanese registry; J-PCI. ${ }^{41}$ This study did not describe patient characteristics at low-volume and high-volume hospitals because the number of variables was limited in the J-PCI registry. On the contrary, the KiCS-PCI registry collects detailed patient and procedural data, which makes our study relevant.

It is further possible that, as indicated by Dimick et $a l,{ }^{42}$ institutional volume could merely be one of the numerous predictors of hospital performance, but perhaps a poor one. Undoubtedly, institutional volume is an important factor, as multiple reports demonstrate that high-volume hospitals exhibit superior performance in high-risk procedures. ${ }^{434}$ However, the magnitude of the volume-outcome relationship has been suggested to be modest in common procedures ${ }^{45-47}$; PCI has indeed become a common procedure in Japan, being performed around 200000 times annually. ${ }^{35}$

Historically, Japanese medical policy has attached the utmost importance to providing accessible, comprehensive medical care with little concern regarding cost. According to the 2015 Organisation for Economic Co-operation and Development (OECD) Health Statistics, the number of hospital beds per capita was highest in Japan, compared with other OECD countries (13.3 beds per 1000 population). Japan further has the highest number of hospitals relative to its population, with 67.3 hospitals per million individuals. Moreover, patients in Japan can access cardiology specialists directly. However, with its rapidly ageing society, adjustments must be made to the current medical system. ${ }^{48}$ In Japan, where population density is relatively high, it may be possible to centralise the treatment of patients with cardiovascular disease within specialised high-volume centres. This centralisation of PCI centres is in fact part of the Japanese policy agenda of specialisation of hospital beds (Improving the Quality of Japan's Hospital Care; OECD Reviews of Healthcare), attempting to provide a possible solution to the rapidly expanding Japanese healthcare budget.

\section{Limitations}

Our study has several limitations. First, this is an observational study and not a randomised trial. Lacking necessary hospital experience, cardiologists in low-volume hospitals may have withheld aggressive PCI for stable patients with complex lesions and other comorbidities in order to avoid complications. Therefore, we cannot discount the possibility that low-volume centres may have sent their sickest patients to other facilities, possibly higher-volume hospitals. This eventuality would be unsurprising: most of the lower-volume hospitals did not have on-site cardiac surgery. However, as we only have data on patients receiving PCI, we cannot estimate the rate of transfer between treatment facilities. Therefore, we cannot rule out the possibility that lower-volume hospitals did not provide revascularisation to patients at increased risk for adverse events. Second, detailed data on angiographic characteristics such as SYNTAX score could not be obtained from the registry. Third, our registry does not include all institutions currently performing PCI in Japan. Fourth, our registry does not include annual operator as a variable and its effect could not be estimated. Recently, a study using data from the NCDR showed an inverse relationship between PCI operator volume and in-hospital mortality. ${ }^{49}$ Finally, we do not present data regarding long-term outcomes of these patient cohorts. However, this warrants further investigation, as a recent report indicated that survival advantage persisted until follow-up in patients receiving treatment at high-performing centres during hospitalisation. ${ }^{50}$

Acknowledgements The authors thank all the investigators, clinical coordinators and institutions involved in the JCD-KiCS study. Investigators: Toshiki Kuno, Hiroyuki Motoda, Yohei Numasawa, Ryota Tabei (Japanese Red Cross Ashikaga Hospital, Ashikaga, Japan); Yutaka Okada (Eiju General Hospital, Tokyo, Japan); Soushin Inoue, Iwao Nakamura (Hino Municipal Hospital, Hino, Japan); Shunsuke Takagi, Takashi Matsubara (Hiratsuka City Hospital, Hiratsuka, Japan); Masashi Takahashi, Keishu Li, Koichiro Sueyoshi (Kawasaki City Municipal Hospital, Kawasaki, Japan); Taku Inohara, Fumiaki Yashima, Atsushi Anzai, Kentaro Hayashida, Takashi Kawakami, Hideaki Kanazawa, Shinsuke Yuasa, Yuichiro Maekawa (Keio University School of Medicine, Tokyo, Japan); Masahiro Suzuki, Keisuke Matsumura (National Hospital Organization Saitama National Hospital, Wako, Japan); Yukinori Ikegami, Jun Fuse, Munehisa Sakamoto, Yukihiko Momiyama (National Hospital Organization Tokyo Medical Center, Tokyo, Japan); Ayaka Endo, Tasuku Hasegawa, Toshiyuki Takahashi, Susumu Nakagawa (Saiseikai Central Hospital, Tokyo, Japan); Takashi Yagi, Kenichiro Shimoji, Shigetaka Noma (Saiseikai Utsunomiya Hospital, Utsunomiya, Japan); Masahito Munakata, Shiro Ishikawa, Takashi Koyama (Saitama City Hospital, Saitama, Japan); Atsushi Mizuno, Yutaro Nishi (St Luke's International Hospital Heart Center, Tokyo, Japan); Daisuke Shinmura, Kazunori Moritani, Masaru Shibata (Tachikawa Kyosai Hospital, Tachikawa, Japan); Kimi Koide, Yoshinori Mano, Takahiro Oki (Tokyo Dental College Ichikawa General Hospital, Ichikawa, Japan); Koji Negishi, Takahiro Koura (Yokohama Municipal Hospital, Yokohama, Japan).

Contributors MK drafted of the manuscript and was involved in conception and design of the study, statistical calculations and analyses, and interpretation of data. SK was involved in conception and design of the study, and revised the manuscript critically for important intellectual content and provided final approval. TK, YN, TO, IN, IU and KF revised the manuscript critically for important intellectual content.

Funding This research was funded by a grant from the Ministry of Education, Culture, Sports, Science and Technology, Japan (KAKENHI No. 21790751, 16H05215, and 16KK0186).

Competing interests Dr. Kohsaka has received grants from Bayer Yakuhin and Daiichi-Sankyo; has received lecture fees from Bayer Yakuhin and Bristol-Myers Squibb.

Patient consent Obtained.

Ethics approval The study was approved by the institutional review board of Keio University School of Medicine. Before the launch of the registry, information on the objectives of the present study, its social significance and an abstract were provided for clinical trial registration with the University Hospital Medical Information Network. This Network is recognised by the International Committee of Medical Journal Editors as an 'acceptable registry', according to a statement issued in September 2004 (UMIN R000005598). The patient record was anonymised and deidentified prior to analysis. Major teaching hospitals within the metropolitan Tokyo area were selected for the pilot phase of this study, and the study protocol was approved by the institutional review board at each site. Patients were enrolled at the event; all the consecutive $\mathrm{PCl}$ procedures during study period, including failure cases, were registered.

Provenance and peer review Not commissioned; externally peer reviewed.

Data sharing statement No additional data are available.

Open access This is an open access article distributed in accordance with the Creative Commons Attribution Non Commercial (CC BY-NC 4.0) license, which permits others to distribute, remix, adapt, build upon this work non-commercially, 
and license their derivative works on different terms, provided the original work is properly cited and the use is non-commercial. See: http://creativecommons.org/ licenses/by-nc/4.0/

(C) Article author(s) (or their employer(s) unless otherwise stated in the text of the article) 2018. All rights reserved. No commercial use is permitted unless otherwise expressly granted.

\section{REFERENCES}

1. Fox KA, Steg PG, Eagle KA, et al. Decline in rates of death and heart failure in acute coronary syndromes, 1999-2006. JAMA 2007;297:1892-900.

2. Luthi JC, McClellan WM, Flanders WD, et al. Variations in the quality of care of patients with acute myocardial infarction among Swiss university hospitals. Int J Qual Health Care 2005;17:229-34.

3. Girotra S, Cram P, Spertus JA, et al. Hospital variation in survival trends for in-hospital cardiac arrest. J Am Heart Assoc 2014;3:e000871.

4. Kontos MC, Rennyson SL, Chen AY, et al. The association of myocardial infarction process of care measures and in-hospital mortality: a report from the NCDR@. Am Heart J 2014;168:766-75.

5. Fitzgibbons RJ, Giobbie-Hurder A, Gibbs JO, et al. Watchful waiting vs repair of inguinal hernia in minimally symptomatic men: a randomized clinical trial. JAMA 2006;295:285-92.

6. Epstein AJ, Yang L, Yang F, et al. A comparison of clinical outcomes from carotid artery stenting among US hospitals. Circ Cardiovasc Qual Outcomes 2014;7:574-80.

7. Badheka AO, Patel NJ, Grover P, et al. Impact of annual operator and institutional volume on percutaneous coronary intervention outcomes: a 5-year United States experience (2005-2009). Circulation 2014;130:1392-406.

8. Zahn R, Gottwik M, Hochadel M, et al. Volume-outcome relation for contemporary percutaneous coronary interventions (PCl) in daily clinical practice: is it limited to high-risk patients? Results from the Registry of Percutaneous Coronary Interventions of the Arbeitsgemeinschaft Leitende Kardiologische Krankenhausarzte (ALKK). Heart 2008;94:329-35.

9. Vakili BA, Kaplan R, Brown DL. Volume-outcome relation for physicians and hospitals performing angioplasty for acute myocardial infarction in New York state. Circulation 2001;104:2171-6.

10. Hannan EL, Wu C, Walford G, et al. Volume-outcome relationships for percutaneous coronary interventions in the stent era. Circulation 2005:112:1171-9.

11. Epstein AJ, Rathore SS, Volpp KG, et al. Hospital percutaneous coronary intervention volume and patient mortality, 1998 to 2000 does the evidence support current procedure volume minimums? $J$ Am Coll Cardiol 2004;43:1755-62.

12. Harold JG, Bass TA, Bashore TM, et al. ACCF/AHA/SCAI 2013 update of the clinical competence statement on coronary artery interventional procedures: a report of the American College of Cardiology Foundation/American Heart Association/American College of Physicians Task Force on Clinical Competence and Training (Writing Committee to Revise the 2007 Clinical Competence Statement on Cardiac Interventional Procedures). J Am Coll Cardiol 2013;62:357-96.

13. Shibuya K, Hashimoto H, Ikegami N, et al. Future of Japan's system of good health at low cost with equity: beyond universal coverage. Lancet 2011;378:1265-73.

14. Onuma $\mathrm{Y}$, Kimura T, Räber L, et al. Differences in coronary risk factors, procedural characteristics, mortality and stent thrombosis between two all-comers percutaneous coronary intervention registries from Europe and Japan: a patient-level data analysis of the Bern-Rotterdam and $\mathrm{j}$-Cypher registries. Eurolntervention 2015;11:533-40.

15. Kohsaka S, Kimura T, Goto M, et al. Difference in patient profiles and outcomes in Japanese versus American patients undergoing coronary revascularization (collaborative study by CREDO-Kyoto and the Texas Heart Institute Research Database). Am J Cardiol 2010;105:1698-704.

16. Cui $\mathrm{R}$, Iso $\mathrm{H}$, Yamagishi $\mathrm{K}$, et al. Trends in the proportions of stroke subtypes and coronary heart disease in the Japanese men and women from 1995 to 2009. Atherosclerosis 2016:248:219-23.

17. Forouhi NG, Sattar N. CVD risk factors and ethnicity-a homogeneous relationship? Atheroscler Suppl 2006;7:11-19.

18. Sekikawa $A$, Satoh $T$, Hayakawa $T$, et al. Coronary heart disease mortality among men aged 35-44 years by prefecture in Japan in 1995-1999 compared with that among white men aged 35-44 by state in the United States in 1995-1998: vital statistics data in recent birth cohort. Jpn Circ J 2001;65:887-92.
19. Lin $\mathrm{HC}$, Lee $\mathrm{HC}$, Chu $\mathrm{CH}$. The volume-outcome relationship of percutaneous coronary intervention: can current procedure volume minimums be applied to a developing country? Am Heart $J$ 2008:155:547-52.

20. Tsuchihashi M, Tsutsui $\mathrm{H}$, Tada $\mathrm{H}$, et al. Volume-outcome relation for hospitals performing angioplasty for acute myocardial infarction: results from the Nationwide Japanese Registry. Circ $J$ 2004;68:887-91.

21. Shiraishi J, Kohno Y, Sawada T, et al. Effects of hospital volume of primary percutaneous coronary interventions on angiographic results and in-hospital outcomes for acute myocardial infarction. Circ $J$ 2008:72:1041-6.

22. Nishigaki K, Yamazaki T, Fukunishi M, et al. Assessment of acute myocardial infarction in Japan by the Japanese Coronary Intervention Study (JCIS) Group. Circ J 2004;68:515-9.

23. Nomura T, Keira N, Kojima A, et al. Effects of cardiologist experience on outcomes of patients with ST-elevated myocardial infarction treated with primary PCl in a local area in Japan. Int Heart $J$ 2011:52:127-30.

24. Weintraub WS, McKay CR, Riner RN, et al. The American College of Cardiology National Database: progress and challenges. American College of Cardiology Database Committee. J Am Coll Cardiol 1997;29:459-65.

25. Brindis RG, Fitzgerald S, Anderson HV, et al. The American College of Cardiology-National Cardiovascular Data Registry (ACC-NCDR): building a national clinical data repository. J Am Coll Cardiol 2001;37:2240-5

26. Ohno $\mathrm{Y}$, Maekawa $\mathrm{Y}$, Miyata $\mathrm{H}$, et al. Impact of periprocedural bleeding on incidence of contrast-induced acute kidney injury in patients treated with percutaneous coronary intervention. J Am Coll Cardiol 2013;62:1260-6.

27. Kodaira M, Miyata $\mathrm{H}$, Numasawa $\mathrm{Y}$, et al. Effect of smoking status on clinical outcome and efficacy of clopidogrel in acute coronary syndrome. Circ J 2016;80:1590-9.

28. Inohara $\mathrm{T}$, Kohsaka $\mathrm{S}$, Miyata $\mathrm{H}$, et al. Appropriateness of coronary interventions in Japan by the US and Japanese standards. Am Heart $J$ 2014;168:854-61.

29. Mehran R, Rao SV, Bhatt DL, et al. Standardized bleeding definitions for cardiovascular clinical trials: a consensus report from the Bleeding Academic Research Consortium. Circulation 2011:123:2736-47.

30. Austin PC, Tu JV, Alter DA. Comparing hierarchical modeling with traditional logistic regression analysis among patients hospitalized with acute myocardial infarction: should we be analyzing cardiovascular outcomes data differently? Am Heart $J$ 2003:145:27-35.

31. Peterson ED, Dai D, DeLong ER, et al. Contemporary mortality risk prediction for percutaneous coronary intervention: results from 588,398 procedures in the National Cardiovascular Data Registry. $J$ Am Coll Cardiol 2010;55:1923-32.

32. Pandey A, McGuire DK, de Lemos JA, et al. Revascularization trends in patients with diabetes mellitus and multivessel coronary artery disease presenting with non-ST elevation myocardial infarction: insights from the National Cardiovascular Data Registry Acute Coronary Treatment and Intervention Outcomes Network RegistryGet With the Guidelines (NCDR ACTION Registry-GWTG). Circ Cardiovasc Qual Outcomes 2016:9:197-205.

33. Bortnick AE, Epps KC, Selzer F, et al. Five-year follow-up of patients treated for coronary artery disease in the face of an increasing burden of co-morbidity and disease complexity (from the NHLBI Dynamic Registry). Am J Cardiol 2014;113:573-9.

34. Lee KS, Kwak JM. Effect of patient risk on the volume-outcome relationship in obstetric delivery services. Health Policy 2014;118:407-12

35. Kohsaka S, Miyata $\mathrm{H}$, Ueda I, et al. An international comparison of patients undergoing percutaneous coronary intervention: a collaborative study of the National Cardiovascular Data Registry (NCDR) and Japan Cardiovascular Database-Keio interhospital Cardiovascular Studies (JCD-KiCS). Am Heart J 2015;170:1077-85.

36. Akin I, Hochadel M, Schneider S, et al. Volume-outcomes relationship in the era of modern coronary intervention-results from the prospective multicenter German DES.DE Registry. Catheter Cardiovasc Interv 2013;82:E788-97.

37. Kumbhani DJ, Fonarow GC, Cannon CP, et al. Temporal trends for secondary prevention measures among patients hospitalized with coronary artery disease. Am J Med 2015;128:426.e1-9.

38. Pocock SJ, Stone GW. The primary outcome is positive-is that good enough? N Engl J Med 2016;375:971-9.

39. Lin X, Tao H, Cai M, et al. A systematic review and meta-analysis of the relationship between hospital volume and the outcomes of percutaneous coronary intervention. Medicine 2016;95:e2687. 
40. Miyata $\mathrm{H}$, Motomura N, Ueda $\mathrm{Y}$, et al. Effect of procedural volume on outcome of coronary artery bypass graft surgery in Japan: implication toward public reporting and minimal volume standards. $J$ Thorac Cardiovasc Surg 2008;135:1306-12.

41. Inohara T, Kohsaka S, Yamaji K, et al. Impact of institutional and operator volume on short-term outcomes of percutaneous coronary intervention: a report from the Japanese Nationwide Registry. JACC Cardiovasc Interv 2017;10:918-27.

42. Dimick JB, Welch HG, Birkmeyer JD. Surgical mortality as an indicator of hospital quality: the problem with small sample size. JAMA 2004;292:847-51.

43. Bach PB, Cramer LD, Schrag D, et al. The influence of hospital volume on survival after resection for lung cancer. $N$ Engl $J$ Med $2001 ; 345: 181-8$

44. Birkmeyer JD, Sun Y, Wong SL, et al. Hospital volume and late survival after cancer surgery. Ann Surg 2007;245:777-83.

45. Neychev VK, Ghanem M, Blackwood SL, et al. Parathyroid surgery can be safely performed in a community hospital by experienced parathyroid surgeons: a retrospective cohort study. Int J Surg 2016;27:72-6.

46. Shackley P, Slack R, Booth A, et al. Is there a positive volumeoutcome relationship in peripheral vascular surgery? Results of a systematic review. Eur J Vasc Endovasc Surg 2000;20:326-35.

47. Kreder HJ, Grosso P, Williams JI, et al. Provider volume and other predictors of outcome after total knee arthroplasty: a population study in Ontario. Can J Surg 2003;46:15-22.

48. Miyata $\mathrm{H}$, Gotoh $\mathrm{M}$, Hashimoto $\mathrm{H}$, et al. Challenges and prospects of a clinical database linked to the board certification system. Surg Today 2014;44:1991-9.

49. Fanaroff $A C$, Zakroysky $P$, Dai $D$, et al. Outcomes of $\mathrm{PCl}$ in relation to procedural characteristics and operator volumes in the United States. J Am Coll Cardiol 2017;69:2913-24.

50. Bucholz EM, Butala NM, Ma S, et al. Life expectancy after myocardial infarction, according to hospital performance. $N$ Engl $J$ Med 2016;375:1332-42. 